\title{
Spectroscopy and production of quarkonia and heavy flavour at ATLAS ***
}

\author{
Semen Turchikhin ${ }^{\mathrm{a}}$, on behalf of the ATLAS Collaboration
}

${ }^{a}$ Joint Institute for Nuclear Research, Dubna, Russia

\begin{abstract}
This proceedings contribution presents recent results of the ATLAS experiment at the LHC on measurements of heavy quarkonium production. These include a study of exotic state $X(3872)$, measurement of prompt $J / \psi$ pair production in $p p$ collisions, and heavy quarkonium production measurements with $p+\mathrm{Pb}$ and $\mathrm{Pb}+\mathrm{Pb}$ collision data.
\end{abstract}

Keywords: charmonium, bottomonium, exotic states, heavy ion collisions

\section{Introduction}

The ATLAS experiment [1] is a general purpose detector at LHC designed for a wide range of particle physics studies. Its physics programme includes heavy flavour studies mainly focusing on final states with two muons, since corresponding triggers have sufficiently low muon thresholds. Heavy quarkonium states decaying to muon pairs represent a good tool for studying heavy quark dynamics in ATLAS.

Four studies are reviewed below. These are measurement of $X(3872)$ exotic state production and of prompt $J / \psi$ pair production in $p p$ collisions, and two measurements of heavy quarkonium production with $\mathrm{Pb}+\mathrm{Pb}$ and $p+\mathrm{Pb}$ collision data.

\section{2. $X(3872)$ production measurement}

The $X(3872)$ particle was discovered by the Belle experiment in 2003 [2] and confirmed by others shortly after. Its mass, narrow width, quantum numbers and decay properties measured in several experiments make it unlikely to be a conventional quarkonium state. There is no final theoretical picture for this particle yet and various models are proposed to describe its structure [3, 4].

${ }^{*}$ Talk given at 22nd International Conference in Quantum Chromodynamics (QCD'19), 2-5 July 2019, Montpellier - FR

${ }^{* *}$ Copyright CERN for the benefit of the ATLAS Collaboration. CC-BY-4.0 license.
ATLAS has studied the production of $X(3872)$ in the decay mode $X(3872) \rightarrow J / \psi \pi^{+} \pi^{-}$, using the decay $\psi(2 \mathrm{~S}) \rightarrow J / \psi \pi^{+} \pi^{-}$as a normalization channel [5]. The analysis is done with the $p p$ collision data collected at $\sqrt{s}=8 \mathrm{TeV}$ corresponding to an integrated luminosity of $11.4 \mathrm{fb}^{-1}$.

The production cross-section is measured in five bins of $p_{\mathrm{T}}$. In order to separate the prompt and non-prompt production, the data in each $p_{\mathrm{T}}$ bin are split into intervals of pseudo-proper lifetime. An attempt to describe the non-prompt component of the signal distribution using a single effective lifetime, $\tau_{\text {eff }}$, results in smaller values of $\tau_{\text {eff }}$ at low $p_{\mathrm{T}}$ for $X(3872)$, while for the $\psi(2 \mathrm{~S})$ it is measured to be uniform across the $p_{\mathrm{T}}$ range, as shown in Figure 1. This possibly indicates a different $X(3872)$ production mechanism at low $p_{\mathrm{T}}$. An alternative model allows for two non-prompt contributions with different effective lifetimes, assuming the short-lived component to originate from the $B_{c}^{+}$meson decays. The fraction of this short-lived component in a range $p_{\mathrm{T}}(X(3872))>10 \mathrm{GeV}$ is measured to be

$$
\begin{gathered}
\frac{\sigma\left(p p \rightarrow B_{c}^{+}+\text {any }\right) \mathcal{B}\left(B_{c}^{+} \rightarrow X(3872)+\text { any }\right)}{\sigma(p p \rightarrow \text { non-prompt } X(3872)+\text { any })}= \\
(25 \pm 13 \text { (stat. }) \pm 2(\text { syst. }) \pm 5(\text { spin })) \% .
\end{gathered}
$$

Since the $B_{c}^{+}$production makes up only a small fraction of the inclusive beauty production, this value of the ratio would mean that the production of $X(3872)$ in $B_{c}^{+}$ 
decays is strongly enhanced compared to its production in the decays of other $b$ hadrons.

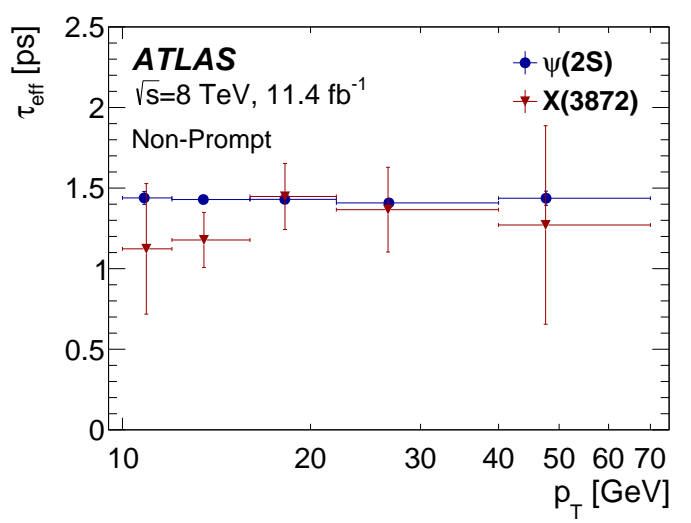

Figure 1: Measured effective pseudo-proper lifetimes in bins of $p_{\mathrm{T}}$ for non-prompt $X(3872)$ and $\psi(2 S)$. Figures are taken from Ref. [5].

Figure 2 shows the measured $X(3872)$ differential production cross-section for prompt and non-prompt production. The prompt production is well described by the predictions based on NLO NRQCD. The FONLL predictions of the non-prompt $\psi(2 \mathrm{~S})$ production, recalculated to $X(3872)$ using the branching fraction extracted from the Tevatron data, significantly overestimate the non-prompt production of $X(3872)$, especially at large transverse momenta.

\section{3. $J / \psi$ pair production}

A $J / \psi$ pair can be produced in $p p$ collisions either by single parton scattering (SPS) or by double parton scattering (DPS) via two independent parton-parton interactions. The study of these processes provides a better understanding of higher order and non-perturbative QCD.

A simplified ansatz for defining the DPS crosssection in terms of the production cross-sections of the two final states and an effective cross-section, $\sigma_{\text {eff }}$, renders for $\mathrm{di}-J / \psi$ production as

$$
\sigma_{\mathrm{eff}}=\frac{1}{2} \frac{\sigma_{J / \psi}^{2}}{\sigma_{\mathrm{DPS}}^{J / \psi, J / \psi}}=\frac{1}{2} \frac{\sigma_{J / \psi}^{2}}{f_{\mathrm{DPS}} \times \sigma_{J / \psi J / \psi}} .
$$

The $\sigma_{\text {eff }}$ is related to the spatial separation between partons inside the proton and is assumed to be independent of energy and of parton interaction process to a firstorder approximation in perturbative QCD predictions.

ATLAS measurement of prompt $J / \psi$ pair production cross-section [6] uses the $p p$ collision data collected at
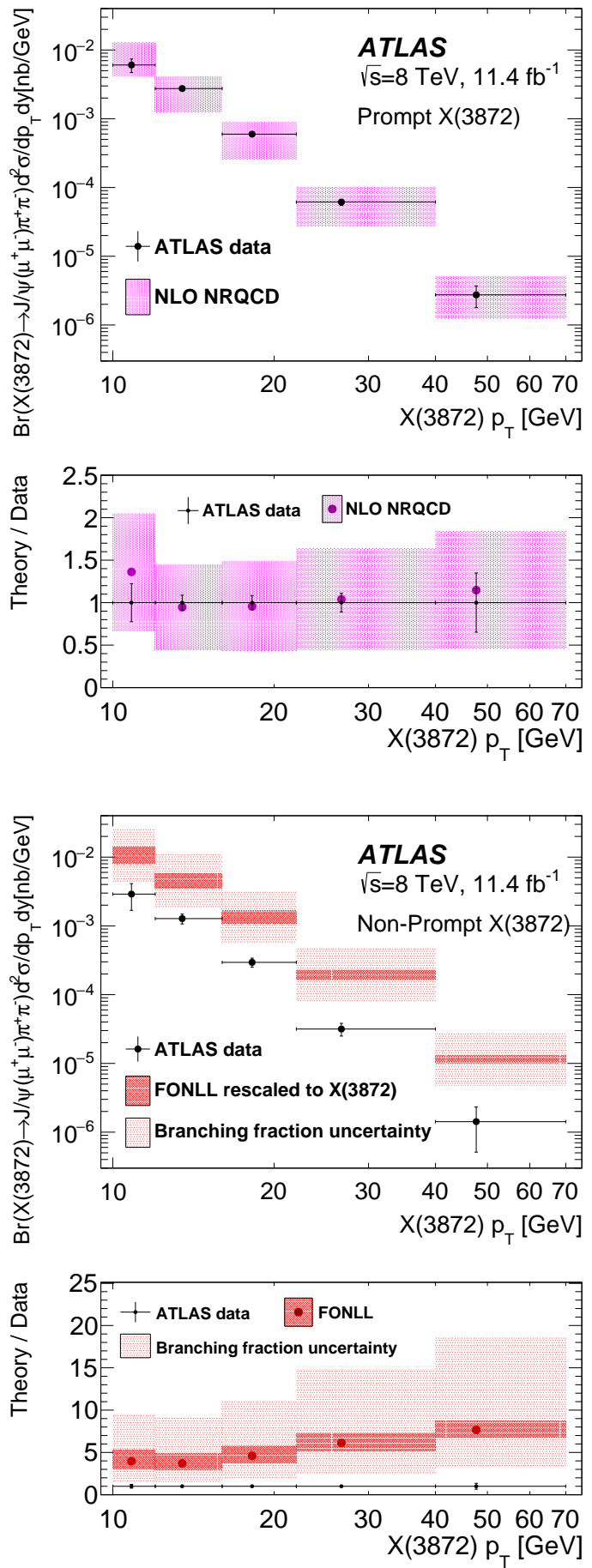

Figure 2: Measured cross-section times branching fraction as a function of $p_{\mathrm{T}}$ for (top) prompt and (bottom) non-prompt $X(3872)$ compared to theoretical expectations. Figures are taken from Ref. [5]. 
$\sqrt{s}=8 \mathrm{TeV}$ corresponding to an integrated luminosity of $11.4 \mathrm{fb}^{-1}$

Using $J / \psi$ reconstructed from two independent events in data as a template for DPS, the fraction of prompt-prompt $J / \psi$ pairs produced via DPS is measured to be $f_{\text {DPS }}=(9.2 \pm 2.1$ (stat.) \pm 0.5 (syst.) $) \%$. The effective cross-section is measured to be $\sigma_{\text {eff }}=$ $6.3 \pm 1.6$ (stat.) \pm 1.0 (syst.) $\pm 0.1(\mathrm{BF}) \pm 0.1$ (lumi.) $\mathrm{mb}$, where the $\mathrm{BF}$ uncertainty corresponds to the uncertainty of $\mathcal{B}\left(J / \psi \rightarrow \mu^{+} \mu^{-}\right)$. Similarly to results of other experiments, this value extracted using di-quarkonium states is lower than those measured for other final states.

Figure 3 shows the differential $J / \psi$ pair production cross-section and the estimated DPS contribution. The data is in a reasonable agreement with the predictions made in leading-order QCD for the DPS and next-toleading-order colour singlet non-relativistic QCD calculations without loops (NLO*) for the SPS contribution.

\section{Heavy quarkonia production in $\mathrm{Pb}+\mathrm{Pb}$ and $p+\mathrm{Pb}$ collisions}

Quarkonia production in ultra-relativistic heavy ion collisions is a valuable probe of the deconfined quarkgluon plasma. A possible suppression of the production can provide information about the temperature and degree of the deconfinement. Enhancement of the production could also appear when e.g. quarkonium is formed due to recombination of $c \bar{c}$ pair from the medium. Studying the non-prompt quarkonia production is also important as it makes it possible to probe the $b$ quark propagation through the medium. The $b$ quark production may be affected by different suppression mechanisms (energy loss by collisional or radiative processes) than the $c \bar{c}$ production (colour screening in the medium).

The ATLAS measurement [7] of $J / \psi$ and $\psi(2 S)$ production in $\mathrm{Pb}+\mathrm{Pb}$ collisions at $\sqrt{s_{\mathrm{NN}}}=5.02 \mathrm{TeV}$ and in $p p$ collisions at $\sqrt{s}=5.02 \mathrm{TeV}$ is done using data collected in 2015 corresponding to integrated luminosities of $0.42 \mathrm{nb}^{-1}$ and $25 \mathrm{pb}^{-1}$, respectively.

Figure 4 shows the nuclear modification factor $R_{\mathrm{AA}}$ for prompt and non-prompt $J / \psi$ production ${ }^{1}$ Strong suppression of prompt and non-prompt charmonia is observed in $\mathrm{Pb}+\mathrm{Pb}$ data. The maximum suppression of prompt and non-prompt $J / \psi$ production by factor $4-5$ is observed for the most central collisions. While the

${ }^{1}$ The nuclear modification factor is defined as $R_{\mathrm{AA}}=$ $N_{\mathrm{AA}} /\left(\left\langle T_{\mathrm{AA}}\right\rangle \times \sigma_{p} p\right)$, where $N_{\mathrm{AA}}$ is the per-event $J / \psi$ yield in the nuclear collisions, $\sigma_{p p}$ is the production cross-section in $p p$ collisions at the same energy, and $\left\langle T_{\mathrm{AA}}\right\rangle$ is the mean nuclear thickness function.
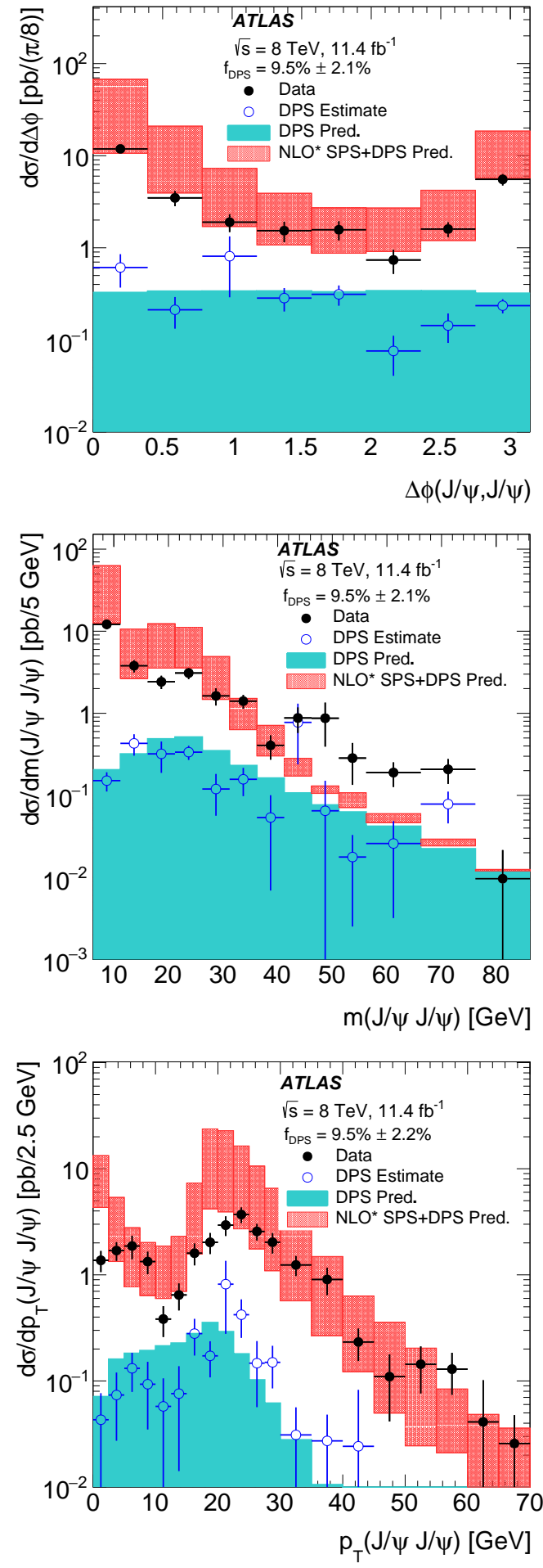

Figure 3: The DPS and the total differential $J / \psi$ pair production crosssection as a function of (top) the azimuthal angle between the two $J / \psi$ mesons, (middle) their invariant mass, and (bottom) the pair $p_{\mathrm{T}}$. Overlaid are the predictions from leading-order DPS and NLO* SPS contributions. Figures are taken from Ref. [6]. 
production of prompt $J / \psi$ mesons is suppressed slightly more in the mid-centrality region, the two classes of meson production have essentially the same pattern. This is not quite expected because the two cases are believed to have rather different physical origins: the non-prompt production should be dominated by propagation of $b$ quarks that extends far outside the deconfined medium, whereas the prompt production happens predominantly within the medium.
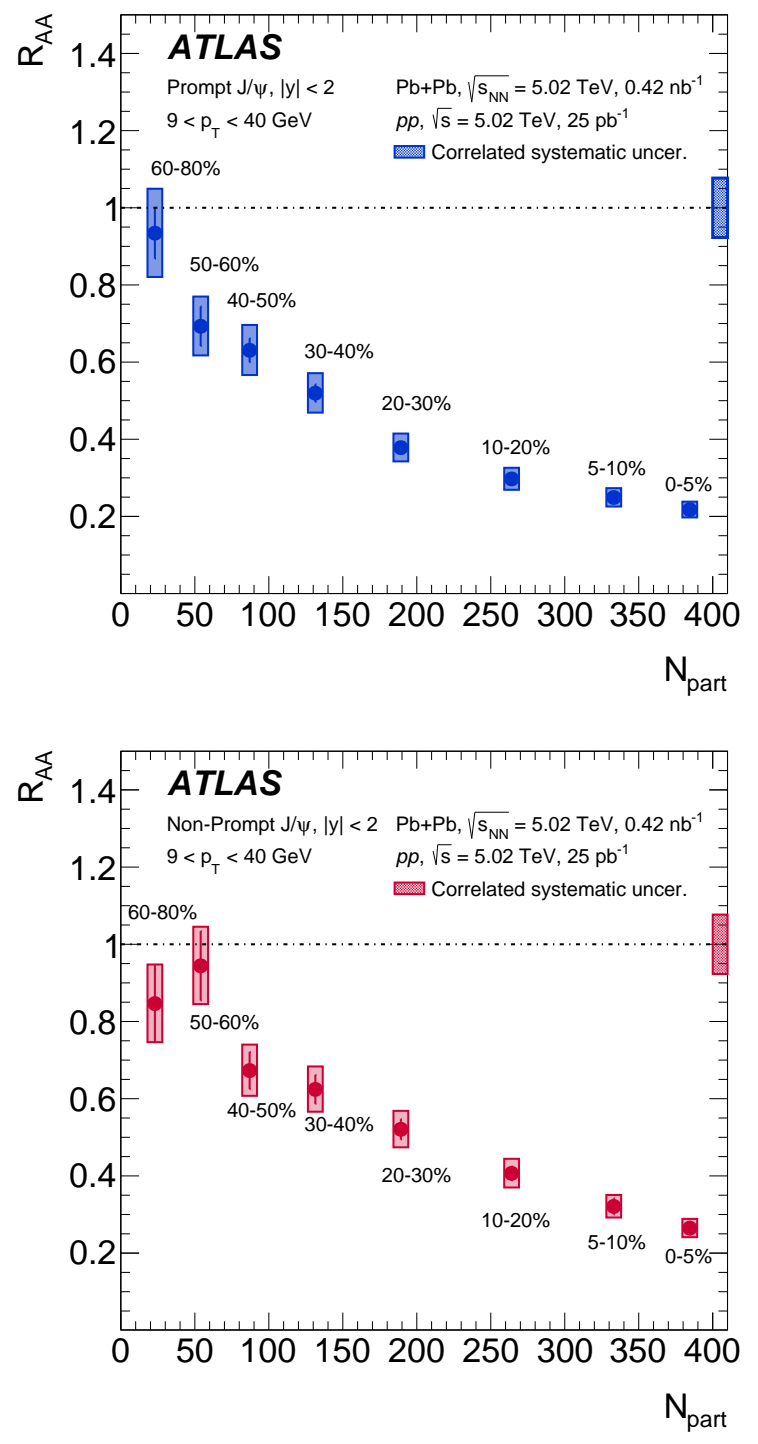

Figure 4: The nuclear modification factor as a function of the number of nucleons participating in a collision, $N_{\text {part }}$, for the (top) prompt and (bottom) non-prompt $J / \psi$. Centrality intervals corresponding to the $N_{\text {part }}$ mean values are shown for each point. Figures are taken from Ref. [7].

Figure 5 shows the $R_{\mathrm{AA}}$ dependence for prompt $J / \psi$ on $p_{\mathrm{T}}$ for the $0-20 \%$ centrality collisions compared with several theoretical models. The data are consistent with the colour screening and colour transparency picture, as well as parton energy-loss description.

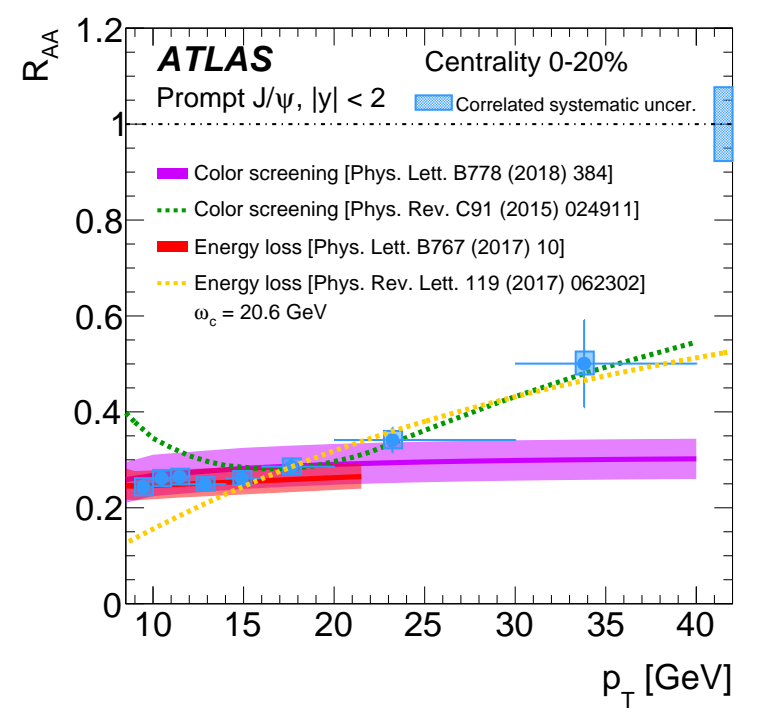

Figure 5: The nuclear modification factor for prompt $J / \psi$ as a function of $p_{\mathrm{T}}$ for $0-20 \%$ centrality collisions, compared with theoretical models. Figures are taken from Ref. [7].

The double ratio of $\psi(2 \mathrm{~S})$ to $J / \psi$ production $\rho_{\mathrm{PbPb}}^{\psi(2 \mathrm{~S}) / J / \psi}=\left(N_{\psi(2 \mathrm{~S})} / N_{J / \psi}\right)_{\mathrm{Pb}+\mathrm{Pb}} /\left(N_{\psi(2 \mathrm{~S})} / N_{J / \psi}\right)_{p p}$ is shown in Figure 6 The ratio is consistent with unity for nonprompt mesons originating from $b$ quarks that lose energy in the medium. For prompt mesons it is different from unity, thus indicating the enhanced suppression of prompt $\psi(2 \mathrm{~S})$ relative to $J / \psi$. This observation is consistent with the interpretation that the $J / \psi$, being a tightly bound quarkonium system, survives the temperature of the hot and dense medium with a higher probability than the more loosely bound $\psi(2 \mathrm{~S})$ state.

Study of heavy quarkonium production in $p+\mathrm{Pb}$ collisions, where a formation of a large region of hot and deconfined matter is not expected, allows to probe Cold Nuclear Matter (CNM) effects. The CNM quarkonium suppression effects can appear either in the initial state (modifications of the nuclear parton distribution functions, parton saturation in the incident nucleus, or parton energy loss through interactions with the nuclear medium), or in final state (absorption of the pairs of heavy quarks).

ATLAS has performed a measurement [8] of the modification of the $J / \psi, \psi(2 \mathrm{~S})$ and $\Upsilon(n S)(n=1,2,3)$ states production in $p+\mathrm{Pb}$ collisions with respect to that in $p p$ collisions using the $p+\mathrm{Pb}$ and $p p$ datasets both 

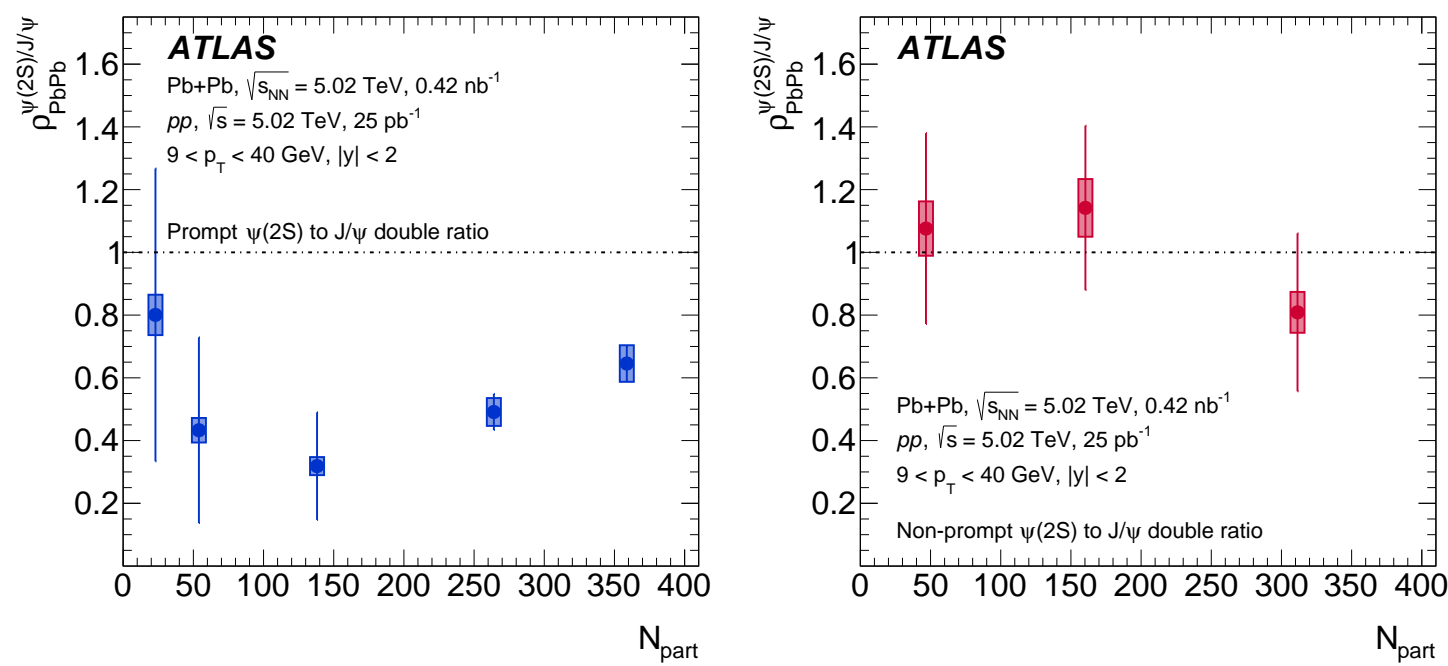

Figure 6: $\psi(2 \mathrm{~S})$ to $J / \psi$ double ratio, as a function of the number of nucleons participating in a collision, $N_{\text {part }}$, for the (left) prompt and (right) non-prompt meson production. Figures are taken from Ref. [7].

collected at a centre-of-mass energy $5.02 \mathrm{TeV}$ and corresponding to integrated luminosities of $28 \mathrm{nb}^{-1}$ and $25 \mathrm{pb}^{-1}$, respectively.

The nuclear modification factor $R_{p \mathrm{~Pb}}=1 / 208$. $\sigma_{p+\mathrm{Pb}} / \sigma_{p p}$ for $\Upsilon(1 \mathrm{~S})$ and $J / \psi$ production as a function of $p_{\mathrm{T}}$ is shown in Figure 7. The $R_{p \mathrm{~Pb}}$ is consistent with unity for both prompt and non-prompt $J / \psi$, while for $\Upsilon(1 \mathrm{~S})$ a suppression at low $p_{\mathrm{T}}$ appears. The latter suggests that the nuclear parton distribution functions are modified relative to those of the nucleon.

Figure 8 shows the double ratios $\rho_{p \mathrm{~Pb}}^{n \mathrm{~S} / 1 \mathrm{~S}}$ as a function of the $p+\mathrm{Pb}$ collision centrality. A suppression of higher states is observed with a significance of $1 \sigma$ for prompt $\psi(2 \mathrm{~S})$ and $2 \sigma$ for $\Upsilon(2,3 \mathrm{~S})$. For $\psi(2 \mathrm{~S})$ to $J / \psi$ and $\Upsilon(2 \mathrm{~S})$ to $\Upsilon(1 \mathrm{~S})$ ratios the suppression increases with centrality, while no conclusion can be made about the $\Upsilon(3 \mathrm{~S})$ to $\Upsilon(1 \mathrm{~S})$ ratio due to high statistical uncertainties. This suggests stronger CNM effects for excited quarkonia compared to that in ground states.

\section{Summary}

ATLAS experiment has a solid programme in heavy flavour physics, largely including studies of charmonium and bottomonium production. Several measurements performed with the Run-1 $p p$ collisions dataset were highlighted in this contribution along with results obtained in $p+\mathrm{Pb}$ and $\mathrm{Pb}+\mathrm{Pb}$ collision data collected during Run 2. These results are compared to theoretical predictions and can serve as probes for the available models.

\section{References}

[1] ATLAS Collaboration, The ATLAS Experiment at the CERN Large Hadron Collider, JINST 3 (2008) S08003. doi : 10.1088/ 1748-0221/3/08/S08003

[2] S. K. Choi, et al., Observation of a narrow charmonium-like state in exclusive $B^{ \pm} \rightarrow K^{ \pm} \pi^{+} \pi^{-} J / \psi$ decays, Phys. Rev. Lett. 91 (2003) 262001. arXiv:hep-ex/0309032 doi:10.1103/ PhysRevLett.91.262001

[3] C. Meng, H. Han, K.-T. Chao, X(3872) and its production at hadron colliders, Phys. Rev. D 96 (2017) 074014. arXiv: 1304.6710 doi:10.1103/PhysRevD.96.074014

[4] A. Tomaradze, S. Dobbs, T. Xiao, K. K. Seth, Precision Measurement of the Mass of the $D^{* 0}$ Meson and the Binding Energy of the $X(3872)$ Meson as a $D^{0} \overline{D^{* 0}}$ Molecule, Phys. Rev. D 91 (2015) 011102. arXiv:1501.01658 doi:10.1103/PhysRevD.91. 011102

[5] ATLAS Collaboration, Measurements of $\psi(2 S)$ and $X(3872) \rightarrow$ $J / \psi \pi^{+} \pi^{-}$production in $p p$ collisions at $\sqrt{s}=8 \mathrm{TeV}$ with the ATLAS detector, JHEP 01 (2017) 117. arXiv:1610.09303. doi : 10.1007/JHEP01(2017) 117

[6] ATLAS Collaboration, Measurement of the prompt $J / \psi$ pair production cross-section in pp collisions at $\sqrt{s}=8 \mathrm{TeV}$ with the ATLAS detector, Eur. Phys. J. C 77 (2017) 76. arXiv : 1612.02950. doi:10.1140/epjc/s10052-017-4644-9

[7] ATLAS Collaboration, Prompt and non-prompt $J / \psi$ and $\psi(2 \mathrm{~S})$ suppression at high transverse momentum in $5.02 \mathrm{TeV} \mathrm{Pb}+\mathrm{Pb}$ collisions with the ATLAS experiment, Eur. Phys. J. C 78 (2018) 762. arXiv:1805.04077, doi:10.1140/epjc/ s10052-018-6219-9

[8] ATLAS Collaboration, Measurement of quarkonium production in proton-lead and proton-proton collisions at $5.02 \mathrm{TeV}$ with the ATLAS detector, Eur. Phys. J. C 78 (2018) 171. arXiv:1709. 03089 doi:10.1140/epjc/s10052-018-5624-4 

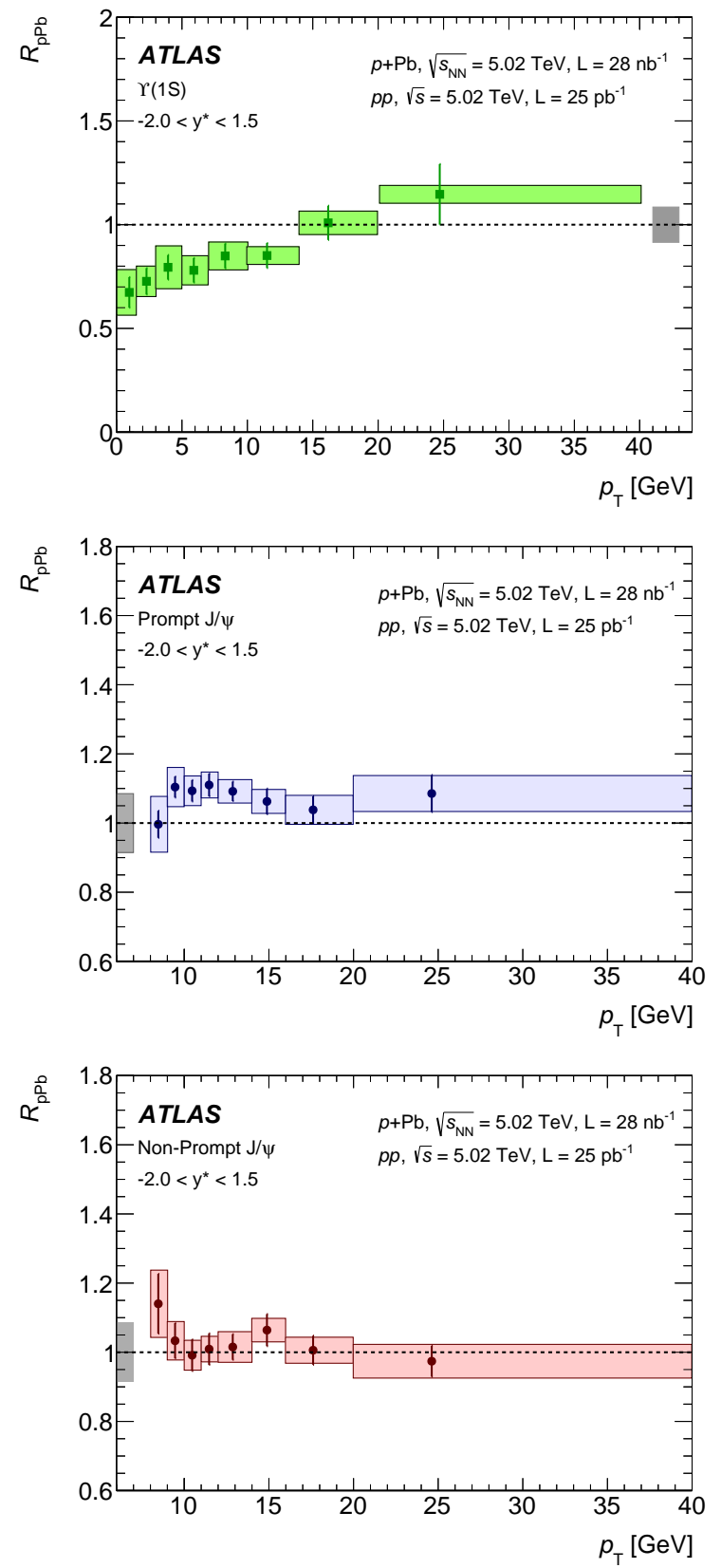

Figure 7: Nuclear modification factor as a function of $p_{\mathrm{T}}$ for (top) $\Upsilon(1 \mathrm{~S})$, (middle) prompt, and (bottom) non-prompt $J / \psi$ production. Figures are taken from Ref. [8].
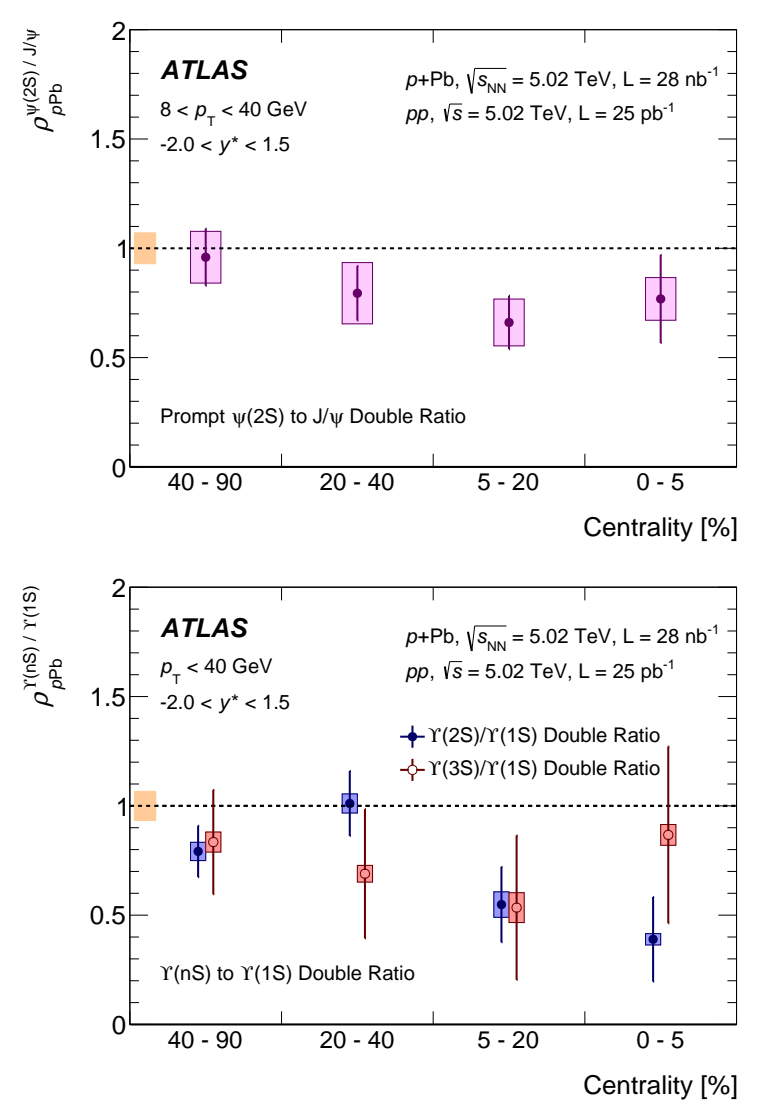

Figure 8: The double ratio $\rho_{p \mathrm{~Pb}}^{n \mathrm{~S} / 1 \mathrm{~S}}$ for (top) prompt charmonium and (bottom) bottomonium as a function of the collision centrality in the kinematic ranges indicated on the plots. The vertical size of the leftmost box corresponds to the total uncertainty of the $p p$ reference. Figures are taken from Ref. [8]. 